\title{
Convexity of Certain Integral Operators Defined by Struve Functions
}

\author{
Shahid Mahmood $\mathbb{D}^{1},{ }^{1}$ Sana Mahroz, ${ }^{2}$ Ayesha Rafiq $\mathbb{D}^{3}{ }^{3}$ \\ Sarfraz Nawaz Malik (iD, ${ }^{2}$ and Mohsan Raza ${ }^{4}{ }^{4}$ \\ ${ }^{1}$ Department of Mechanical Engineering, Sarhad University of Science and Information Technology, Ring Road, Peshawar, Pakistan \\ ${ }^{2}$ COMSATS Institute of Information Technology, Wah Cantt, Pakistan \\ ${ }^{3}$ Institute of Space Technology, Islamabad, Pakistan \\ ${ }^{4}$ Department of Mathematics, Government College University, Faisalabad, Pakistan
}

Correspondence should be addressed to Sarfraz Nawaz Malik; snmalik110@yahoo.com

Received 4 December 2017; Accepted 28 January 2018; Published 1 March 2018

Academic Editor: Henryk Hudzik

Copyright (C) 2018 Shahid Mahmood et al. This is an open access article distributed under the Creative Commons Attribution License, which permits unrestricted use, distribution, and reproduction in any medium, provided the original work is properly cited.

This article deals with some functional inequalities involving Struve function, generalized Struve function, and modified Struve functions. We aim to find the convexity of the integral operator defined by Struve function, generalized Struve function, and modified Struve functions.

\section{Introduction}

We denote by $\mathscr{A}$ the class of functions $f$ which are analytic in the open unit disc $\mathcal{U}=\{z:|z|<1\}$ and of the form

$$
f(z)=z+\sum_{n=2}^{\infty} a_{n} z^{n}
$$

Let $\mathcal{S}$ denote the class of all functions in $\mathscr{A}$ which are univalent in $\mathscr{U}$. Also let $\mathcal{S}^{*}$ and $\mathscr{C}$ be the subclasses of $\mathcal{S}$ consisting of all functions which map $\mathcal{U}$ onto a star shaped with respect to origin and convex domains, respectively. These classes are defined as

$$
\begin{aligned}
\mathcal{S}^{*} & =\left\{f: f \in \mathscr{A}, \operatorname{Re} \frac{z f^{\prime}(z)}{f(z)}>0, z \in \mathcal{U}\right\}, \\
\mathscr{C} & =\left\{f: f \in \mathscr{A}, \operatorname{Re}\left(1+\frac{z f^{\prime \prime}(z)}{f^{\prime}(z)}\right)>0, z \in \mathcal{U}\right\} .
\end{aligned}
$$

Ozaki [1] showed that if a function $f$ is analytic in $\mathcal{U}$ and of the form (1) such that $f(z) f^{\prime}(z) / z \neq 0$ and if either

$$
\mathfrak{R}\left(1+\frac{z f^{\prime \prime}(z)}{f^{\prime}(z)}\right) \geq-\frac{1}{2}, \quad z \in \mathcal{U}
$$

or

$$
\Re\left(1+\frac{z f^{\prime \prime}(z)}{f^{\prime}(z)}\right) \leq \frac{3}{2}, \quad z \in \mathcal{U},
$$

then $f$ is univalent and convex in at least one direction in $\mathcal{U}$. It shows that the constants $-1 / 2$ and $3 / 2$ are, in a certain sense, the best possible constants. In [2], for $\in \mathbb{R}$, consider the class $\mathscr{G}(\beta)$ consisting of locally univalent functions $f \in \mathscr{A}$ that satisfy the condition $\Re\left(1+z f^{\prime \prime}(z) / f^{\prime}(z)\right)<1+\beta / 2$ for $0<\beta \leq 1$. That is,

$$
\begin{aligned}
& \mathscr{G}(\beta) \\
& =\left\{f \in \mathscr{A}: \mathfrak{R}\left(1+\frac{z f^{\prime \prime}(z)}{f^{\prime}(z)}\right)<1+\frac{\beta}{2}, 0<\beta \leq 1\right\} .
\end{aligned}
$$

It can be seen that $\mathscr{G}(\beta) \neq \varnothing$ if and only if $\beta>0$. Since $\mathscr{G}\left(\beta_{1}\right) \subset \mathscr{G}\left(\beta_{2}\right)$ whenever $\beta_{1}<\beta_{2}$, it readily follows that the class $\mathscr{G}(\beta)$ is in $\mathcal{S}^{*}$, for $\beta \in(0,1]$, so in particular, the functions in $\mathscr{G}(\beta)$ are univalent functions. For some more detail about these conditions, see $[3,4]$.

Now we consider the second-order inhomogeneous differential equation 


$$
\begin{gathered}
z^{2} w^{\prime \prime}(z)+z w^{\prime}(z)-\left(z^{2}+v^{2}\right) w(z) \\
=\frac{4(z / 2)^{v+1}}{\sqrt{\pi} \Gamma(v+1 / 2)} .
\end{gathered}
$$

The solution of the homogeneous part is Bessel functions of order $v$, where $v$ is real or complex number. For some more details, see [5]. The particular solution of the inhomogeneous equation defined in (6) is called the Struve function of order $v$. It is defined as

$$
K_{v}(z)=\sum_{n=0}^{\infty} \frac{(-1)^{n}(z / 2)^{2 n+v+1}}{\Gamma(n+3 / 2) \Gamma(v+n+3 / 2)} .
$$

Now we consider the differential equation

$$
\begin{gathered}
z^{2} w^{\prime \prime}(z)+z w^{\prime}(z)-\left(z^{2}+v^{2}\right) w(z) \\
=\frac{4(z / 2)^{v+1}}{\sqrt{\pi} \Gamma(v+1 / 2)}
\end{gathered}
$$

Equation (8) differs from (6) in the coefficients of $w$. Its particular solution is called the modified Struve functions of order $v$ and is given by

$$
\begin{aligned}
L_{v}(z) & =-i e^{-i v \pi / 2} K_{v}(i z) \\
& =\sum_{n=0}^{\infty} \frac{(z / 2)^{2 n+v+1}}{\Gamma(n+3 / 2) \Gamma(v+n+3 / 2)} .
\end{aligned}
$$

Again consider the second-order in-homogenous differential equation

$$
\begin{aligned}
& z^{2} w^{\prime \prime}(z)+b z w^{\prime}(z)+\left[c z^{2}-v^{2}+(1-b) v\right] w(z) \\
& \quad=\frac{4(z / 2)^{v+1}}{\sqrt{\pi} \Gamma(v+b / 2)}
\end{aligned}
$$

where $b, c, v \in \mathbb{C}$. Equation (10) generalizes (6) and (8). In particular for $b=1, c=1$, we obtain (6) and for $b=1, c=$ -1 , we obtain (8). Its particular solution has the series form

$$
w_{v, b, c}(z)=\sum_{n=0}^{\infty} \frac{(-1)^{n} c^{n}(z / 2)^{2 n+v+1}}{\Gamma(n+3 / 2) \Gamma(v+n+(b+2) / 2)}
$$

and is called the generalized Struve function of order $v$. This series is convergent everywhere. We take the transformation

$$
\begin{aligned}
u_{v, b, c}(z) & =2^{v} \sqrt{\pi} \Gamma(v+(b+2) / 2) z^{(-v-1) / 2} w_{v, b, c}(\sqrt{z}) \\
& =\sum_{n=0}^{\infty} \frac{(-c / 4)^{n} z^{n}}{(3 / 2)_{n}(l)_{n}}
\end{aligned}
$$

where $l=v+(b+2) / 2 \neq 0,-1,-2, \ldots$ and $(\gamma)_{n}=\Gamma(\gamma+$ $n) / \Gamma(\gamma)=\gamma(\gamma+1) \cdots(\gamma+n-1)$, where $\Gamma(\cdot)$ denotes the Gamma function. The function $u_{v, b, c}$ is analytic in the whole complex plane and satisfies the differential equation

$$
\begin{aligned}
& 4 z^{2} w^{\prime \prime}(z)+2(2 p+b+3) z w^{\prime}(z) \\
& +[c z+2 p+b] w(z)=2 p+b .
\end{aligned}
$$

The function $u_{v, b, c}$ unifies the Struve functions and modified Struve functions.

The function $u_{v, b, c}$ was introduced and studied by Orhan and Yagmur [6] and further investigated by [7-9]. In last few years, many mathematicians have set the univalence criteria of several those integral operators which preserve the class $\mathcal{S}$. By using a variety of different analytic techniques, operators, and special functions, several authors have studied univalence criterion. Recently Din et al. [10] studied the univalence of integral operators involving generalized Struve functions. These operators are defined as follows:

$$
\begin{aligned}
& \mathscr{F}_{\alpha_{1}, \ldots, \alpha_{n}, \beta}(z)=\left[\beta \int_{0}^{z} t^{\beta-1} \prod_{i=1}^{n}\left(\frac{v_{l_{i}, b, c}(t)}{t}\right)^{1 / \alpha_{i}} d t\right]^{1 / \beta}, \\
& \mathscr{X}_{n, \gamma}(z)=\left[(n \gamma+1) \int_{0}^{z} \prod_{i=1}^{n}\left\{v_{l_{i}, b, c}(t)\right\}^{\gamma} d t\right]^{1 /(n \gamma+1)}, \\
& \mathscr{X}_{\lambda}(z)=\left[\lambda \int_{0}^{Z} t^{\lambda-1}\left(e^{v_{l_{i}, b, c}(t)}\right)^{\lambda} d t\right]^{1 / \lambda} .
\end{aligned}
$$

In this paper, our main aim is to study the convexity and univalence of the integral operators

$$
\begin{aligned}
& W_{v_{i}, b, c, \gamma_{i}}(z)=\int_{0}^{z} \prod_{i=1}^{n}\left(\frac{u_{v_{i}, b, c}(t)}{t}\right)^{\gamma_{i}} d t \\
& G_{v_{i}, b, c, \gamma_{i}}(z)=\int_{0}^{z} \prod_{i=1}^{n}\left(u_{v_{i}, b, c}^{\prime}(t)\right)^{\gamma_{i}} d t .
\end{aligned}
$$

Applications of Struve functions occur in water wave and surface wave problems, unsteady aerodynamics, resistive MHD instability theory, and optical diffraction. More recently, Struve functions appeared in particle quantum dynamic studies of spin decoherence and nanotubes electrodynamics, potential theory, and optics. For more details, we refer to see [11].

Lemma 1 (see [6]). If $b, v \in \mathbb{R}$ and $c \in \mathbb{C}, l=v+(b+2) / 2$ are so constrained that $l>\max \{0,7|c| / 24\}$, then the function $u_{v, b, c}: \mathcal{U} \rightarrow \mathbb{C}$ satisfies the following inequalities:

$$
\begin{aligned}
& \text { (i) }\left|z u_{v, b, c}^{\prime}(z) / u_{v, b, c}(z)-1\right| \leq|c|(6 l-|c|) / 3(4 l-|c|)(3 l-|c|), \\
& \text { (ii) }\left|z u_{v, b, c}^{\prime \prime}(z) / u_{v, b, c}^{\prime}(z)\right| \leq 6|c| /(12 l-7|c|) .
\end{aligned}
$$

\section{Main Results}

In this section, we find the convexity of these integral operators defined by generalized Struve functions, by using above lemma and the following inequalities. We also use Ozaki's condition for the univalence of these operators. 


\subsection{Convexity Criteria for Integral Operators}

Theorem 2. Let $v_{1}, \ldots, v_{n}, b \in \mathbb{R}$ and $c \in \mathbb{C}$ be so constrained that $v_{i}>7|c| / 24$, where $l_{i}=v_{i}+(b+2) / 2(i=1,2,3, \ldots, n)$. Consider the function $u_{v_{i}, b, c}: \mathscr{U} \rightarrow \mathbb{C}$, defined as

$$
u_{v_{i}, b, c}(z)=2^{v_{i}} \sqrt{\pi} \Gamma\left(v_{i}+\frac{b+2}{2}\right) z^{\left(\left(-1-v_{i}\right) / 2\right)} w_{v_{i}, b, c}(z)
$$

Also suppose that $l=\min \left\{l_{1}, l_{2}, \ldots, l_{n}\right\}$ and $\gamma_{1}, \gamma_{2}, \ldots, \gamma_{n}$ be positive real number and that satisfies the inequality

$$
0 \leq 1-\frac{|c|(6 l-|c|)}{3(4 l-|c|)(3 l-|c|)} \sum_{i=1}^{n} \gamma_{i}<1,
$$

then $W_{v_{i}, b, c, \gamma_{i}}(z): \mathscr{U} \rightarrow \mathbb{C}$ defined in (15) is in class $\mathscr{C}(\alpha)$ and

$$
\alpha=1-\frac{|c|(6 l-|c|)}{3(4 l-|c|)(3 l-|c|)} \sum_{i=1}^{n} \gamma_{i} .
$$

Proof. It is clear that $u_{v_{i}, b, c} \in \mathscr{A}$ as it satisfies the condition

$$
u_{v_{i}, b, c}(0)=u_{v_{i}, b, c}^{\prime}-1=0 \text {. }
$$

Differentiating (15), we obtain

$$
W_{v_{i}, b, c, \gamma_{i}}^{\prime}(z)=\prod_{i=1}^{n}\left(\frac{u_{v_{i}, b, c}(z)}{z}\right)^{\gamma_{i}} .
$$

Clearly

$$
W_{v_{i}, b, c, \gamma_{i}}(0)=W_{v_{i}, b, c, \gamma_{i}}^{\prime}(0)-1=0 .
$$

Differentiating logarithmically and after simple calculations, it follows that

$$
\begin{aligned}
& \Re\left(1+\frac{z W_{v_{i}, b, c, r_{i}}^{\prime \prime}(z)}{W_{v_{i}, b, c, \gamma_{i}}^{\prime}(z)}\right) \\
& =\sum_{i=1}^{n} \gamma_{i} \mathfrak{R}\left(\frac{z u_{v_{i}, b, c}^{\prime}(z)}{u_{v_{i}, b, c}(z)}\right)+\left(\sum_{i=1}^{n} \gamma_{i}\right) .
\end{aligned}
$$

Using inequality (i) of Lemma 1 , it is easy to see that

$$
\left|\frac{z u_{v, b, c}^{\prime}(z)}{u_{v, b, c}(z)}-1\right| \leq \frac{|c|(6 l-|c|)}{3(4 l-|c|)(3 l-|c|)}
$$

so

$$
\Re\left\{\frac{z u_{v, b, c}^{\prime}(z)}{u_{v, b, c}(z)}\right\}>1-\frac{|c|(6 l-|c|)}{3(4 l-|c|)(3 l-|c|)} .
$$

By putting the above inequality in (23), we have

$$
\begin{aligned}
& \Re\left(1+\frac{z W_{v_{i}, b, c, r_{i}}^{\prime \prime}(z)}{W_{v_{i}, b, c, \gamma_{i}}^{\prime}(z)}\right) \\
& >\sum_{i=1}^{n} \gamma_{i}\left(1-\frac{|c|\left(6 l_{i}-|c|\right)}{3\left(4 l_{i}-|c|\right)\left(3 l_{i}-|c|\right)}\right) \\
& \quad+\left(1-\sum_{i=1}^{n} \gamma_{i}\right) \\
& =\sum_{i=1}^{n} \gamma_{i}-\sum_{i=1}^{n} \gamma_{i}\left(\frac{|c|\left(6 l_{i}-|c|\right)}{3\left(4 l_{i}-|c|\right)\left(3 l_{i}-|c|\right)}\right)+1-\sum_{i=1}^{n} \gamma_{i} \\
& >1-\sum_{i=1}^{n} \gamma_{i}\left(\frac{|c|\left(6 l_{i}-|c|\right)}{3\left(4 l_{i}-|c|\right)\left(3 l_{i}-|c|\right)}\right) .
\end{aligned}
$$

For $z \in \mathcal{U}$ and $l_{i}=v_{i}+(b+2) / 2>(7|c| / 24), \forall i=1, \ldots, n$, we consider that the function

$$
\Omega:\left(\frac{7|c|}{24}, \infty\right) \rightarrow \mathbb{R}
$$

defined by

$$
\Omega(x)=\frac{|c|(6 x-|c|)}{3(4 x-|c|)(3 x-|c|)},
$$

is decreasing $\forall i=1, \ldots, n$, and therefore

$$
\frac{|c|\left(6 l_{i}-|c|\right)}{3\left(4 l_{i}-|c|\right)\left(3 l_{i}-|c|\right)} \leq \frac{|c|(6 l-|c|)}{3(4 l-|c|)(3 l-|c|)}
$$

Hence

$$
\begin{aligned}
& \Re\left(1+\frac{z W_{v_{i}, b, c, r_{i}}^{\prime \prime}(z)}{W_{v_{i}, b, c, \gamma_{i}}^{\prime}(z)}\right) \\
& \quad>1-\frac{|c|(6 l-|c|)}{3(4 l-|c|)(3 l-|c|)} \sum_{i=1}^{n} \gamma_{i},
\end{aligned}
$$

which completes the proof that $W_{v_{i}, b, c, \gamma_{i}}(z) \in \mathscr{C}(\alpha)$.

Theorem 3. Let $v_{1}, \ldots, v_{n}, b \in \mathbb{R}$ and $c \in \mathbb{C}$ be so constrained that $l_{i}>7|c| / 24$ where $l_{i}=v_{i}+(b+2) / 2(i=1,2,3, \ldots, n)$. Consider the function $u_{v_{i}, b, c}: \mathscr{U} \rightarrow \mathbb{C}$, defined as

$$
u_{v_{i}, b, c}(z)=2^{v_{i}} \sqrt{\pi} \Gamma\left(v_{i}+\frac{b+2}{2}\right) z^{\left(\left(-1-v_{i}\right) / 2\right)} w_{v_{i}, b, c}(z) .
$$

Also suppose that $l=\min \left\{l_{1}, l_{2}, \ldots, l_{n}\right\}$ and $\gamma_{1}, \gamma_{2}, \ldots, \gamma_{n}$ be positive real number and that satisfies the inequality

$$
0 \leq 1-\frac{6|c|}{12 l-7|c|} \sum_{i=1}^{n} \gamma_{i}<1,
$$

then $G_{v_{i}, b, c, \gamma_{i}}(z): \mathscr{U} \rightarrow \mathbb{C}$ defined in (16) is in class $\mathscr{C}(\alpha)$, where

$$
\alpha=1-\frac{6|c|}{12 l-7|c|} \sum_{i=1}^{n} \gamma_{i}
$$


Proof. Differentiating (16), we obtain

$$
G_{v_{i}, b, c, \gamma_{i}}^{\prime}(z)=\prod_{i=1}^{n}\left(u_{v_{i}, b, c}^{\prime}(z)\right)^{\gamma_{i}} .
$$

Clearly

$$
G_{v_{i}, b, c, \gamma_{i}}(0)=G_{v_{i}, b, c, \gamma_{i}}^{\prime}(0)-1=0 .
$$

Differentiating logarithmically and after simple calculations, we obtain

$$
\mathfrak{R}\left(1+\frac{z G_{v_{i}, b, c, r_{i}}^{\prime \prime}(z)}{G_{v_{i}, b, c, \gamma_{i}}^{\prime}(z)}\right)=\sum_{i=1}^{n} \gamma_{i} \mathfrak{R}\left(\frac{z l_{v_{i}, b, c}^{\prime \prime}(z)}{l_{v_{i}, b, c}^{\prime}(z)}\right)+1 .
$$

By using inequality (ii) of Lemma 1, we have

$$
\left|\frac{z u_{v_{i}, b, c}^{\prime \prime}(z)}{u_{v_{i}, b, c}^{\prime}(z)}\right| \leq \frac{6|c|}{12 l-7|c|}
$$

so

$$
\Re\left\{\frac{z u_{v_{i}, b, c}^{\prime \prime}(z)}{u_{v_{i}, b, c}^{\prime}(z)}\right\}>-\frac{6|c|}{12 l-7|c|} .
$$

Using above relation in (36), we have

$$
\begin{aligned}
& \Re\left(1+\frac{z G_{v_{i}, b, c, r_{i}}^{\prime \prime}(z)}{G_{v_{i}, b, c, \gamma_{i}}^{\prime}(z)}\right)>\sum_{i=1}^{n} \gamma_{i}\left(-\frac{6|c|}{12 l_{i}-7|c|}\right)+1 \\
& \quad=1-\sum_{i=1}^{n} \gamma_{i}\left(\frac{6|c|}{12 l_{i}-7|c|}\right) .
\end{aligned}
$$

For $z \in \mathcal{U}$ and $l_{i}=v_{i}+(b+2) / 2>(7|c| / 24)(i=1, \ldots, n)$,we consider that the function

$$
\Psi:\left(\frac{7|c|}{24}, \infty\right) \rightarrow \mathbb{R}
$$

defined by

$$
\Psi(x)=\frac{6|c|}{12 x-7|c|}
$$

is decreasing $\forall i=1, \ldots, n$, and therefore

$$
\frac{6|c|}{12 l_{i}-7|c|} \leq \frac{6|c|}{12 l-7|c|}
$$

Hence

$$
\Re\left(1+\frac{z G_{v_{i}, b, c, r_{i}}^{\prime \prime}(z)}{G_{v_{i}, b, c, \gamma_{i}}^{\prime}(z)}\right)>1-\sum_{i=1}^{n} \gamma_{i} \frac{6|c|}{12 l-7|c|} .
$$

We have $\alpha=1-(6|c| /(12 l-7|c|)) \sum_{i=1}^{n} \gamma_{i}$ which completes the proof that $G_{v_{i}, b, c, \gamma_{i}}(z) \in \mathscr{C}(\alpha)$.

\section{Some Special Cases of Struve Functions and Modified Struve Functions}

3.1. Struve Functions. By setting $b=c=1$ in (11), we obtain the Struve function of first kind of order $v$, denoted by $K_{v}(z)$ defined in (7). Let $\mathscr{K}_{v}: \mathcal{U} \rightarrow \mathbb{C}$ be defined as

$$
\mathscr{K}_{v}(z)=2^{v} \sqrt{\pi} \Gamma\left(v+\frac{b+2}{2}\right) z^{(-1-v) / 2} K_{v}(\sqrt{z}) .
$$

We observe that

$$
\begin{aligned}
\mathscr{K}_{-1 / 2}(z) & =\sqrt{z} \sin (\sqrt{z}), \\
\mathscr{K}_{1 / 2}(z) & =2(1-\cos \sqrt{z}), \\
\mathscr{K}_{3 / 2}(z) & =4\left(1+\frac{2}{z}\right)-8\left(\frac{\sin (\sqrt{z})}{\sqrt{z}}+\frac{\cos (\sqrt{z})}{\sqrt{z}}\right) .
\end{aligned}
$$

Corollary 4. Let $v_{1}, \ldots, v_{n}>-1.75$, where $n \in \mathbb{N}$. Consider the function $\mathscr{K}_{v}(z): \mathcal{U} \rightarrow \mathbb{C}$ defined by

$$
\mathscr{K}_{v_{i}}(z)=2^{v_{i}} \sqrt{\pi} \Gamma\left(v_{i}+\frac{3}{2}\right) z^{\left(\left(-v_{i}-1\right) / 2\right)} K_{v_{i}}(\sqrt{z}) .
$$

Also let $v=\min \left\{v_{1}, v_{2}, \ldots, v_{n}\right\}$ and $\gamma_{1}, \gamma_{2}, \ldots, \gamma_{n}$ be positive real numbers. Suppose that these numbers satisfy the following inequality.

$$
0 \leq 1-\frac{4(3 v+4)}{3\left(24 v^{2}+58 v+35\right)} \sum_{i=1}^{n} \gamma_{i}<1,
$$

then the function $W_{v_{i}, \gamma_{i}}(z): \mathcal{U} \rightarrow \mathbb{C}$ defined as

$$
W_{v_{i}, \gamma_{i}}(z)=\int_{0}^{z} \prod_{i=1}^{n}\left(\frac{\mathscr{K}_{v_{i}}(t)}{t}\right)^{\gamma_{i}} d t
$$

is in class $\mathscr{C}(\alpha)$, where

$$
\alpha=1-\frac{4(3 v+4)}{3\left(24 v^{2}+58 v+35\right)} \sum_{i=1}^{n} \gamma_{i} .
$$

In particular

(i) First at $v=-1 / 2$, the function

$$
\begin{aligned}
W_{-1 / 2, \gamma}(z) & =\int_{0}^{z}\left(\frac{\mathscr{K}_{-1 / 2}(t)}{t}\right)^{\gamma} d t \\
& =\int_{0}^{z}\left(\frac{\sin (\sqrt{t})}{\sqrt{t}}\right)^{\gamma} d t
\end{aligned}
$$

is in class $\mathscr{C}\left(\alpha_{1}\right)$ for $\gamma \leq 18 / 5$, where

$$
\alpha_{1}=1-\frac{5}{18} \gamma
$$

(ii) For $v=1 / 2$, then the function

$$
\begin{aligned}
W_{1 / 2, \gamma}(z) & =\int_{0}^{z}\left(\frac{\mathscr{K}_{1 / 2}(t)}{t}\right)^{\gamma} d t \\
& =\int_{0}^{z}\left(\frac{2(1-\cos \sqrt{t})}{t}\right)^{\gamma} d t
\end{aligned}
$$

is in class $\mathscr{C}\left(\alpha_{2}\right)$ for $\gamma \leq 105 / 11$, where $\alpha_{2}=1-(11 / 105) \gamma$. 
(iii) For $v=3 / 2$, the function

$$
\begin{aligned}
& W_{3 / 2, \gamma}(z)=\int_{0}^{z}\left(\frac{\mathscr{K}_{3 / 2}(t)}{t}\right)^{\gamma} d t \\
& =\int_{0}^{z}\left(\frac{4(1+2 / t)-8(\sin (\sqrt{t}) / \sqrt{t}+\cos (\sqrt{t}) / \sqrt{t})}{t}\right)^{\gamma} d t
\end{aligned}
$$

is in class $\mathscr{C}\left(\alpha_{3}\right)$ for $\gamma \leq 264 / 17$, where $\alpha_{3}=1-(17 / 264) \gamma$.

Corollary 5. Let $v_{1}, \ldots, v_{n}>-1.75$ where $n \in \mathbb{N}$. Consider the function $\mathscr{K}_{v}(z): \mathcal{U} \rightarrow \mathbb{C}$ defined by (46) and $v=$ $\min \left\{v_{1}, v_{2}, \ldots, v_{n}\right\}$ and $\gamma_{1}, \gamma_{2}, \ldots, \gamma_{n}$ be positive real numbers such that these numbers satisfy the inequality

$$
0 \leq 1-\frac{6}{12 v+11} \sum_{i=1}^{n} \gamma_{i}<1,
$$

then the function $G_{v_{i}, \gamma_{i}}(z): \mathscr{U} \rightarrow \mathbb{C}$ defined as

$$
G_{v_{i}, \gamma_{i}}(z)=\int_{0}^{z} \prod_{i=1}^{n}\left(\mathscr{K}_{t_{i}}^{\prime}(t)\right)^{\gamma_{i}} d t
$$

is in class $C(\alpha)$, where $0 \leq \alpha<1$ and

$$
\alpha=1-\frac{6}{12 v+11} \sum_{i=1}^{n} \gamma_{i}
$$

In particular, one has the following:

(i) For $v=-1 / 2$, then

$$
\begin{aligned}
G_{-1 / 2, \gamma}(z) & =\int_{0}^{z}\left(\mathscr{K}_{-1 / 2}^{\prime}(t)\right)^{\gamma} d t \\
& =\int_{0}^{z}\left(\frac{1}{2 \sqrt{t}} \sin (\sqrt{t})+\frac{\cos (\sqrt{t})}{2}\right)^{\gamma} d t
\end{aligned}
$$

is in class $\mathscr{C}\left(\alpha_{1}\right)$ for $\gamma \leq 5 / 6$, where $\alpha_{1}=1-(6 / 5) \gamma$.

(ii) For $v=1 / 2$, the function

$$
\begin{aligned}
& G_{1 / 2, \gamma}(z)=\int_{0}^{z}\left(\mathscr{K}_{1 / 2}^{\prime}(t)\right)^{\gamma} d t \\
& G_{1 / 2, \gamma}(z)=\int_{0}^{z}\left(\frac{\sin (\sqrt{t})}{\sqrt{t}}\right)^{\gamma} d t
\end{aligned}
$$

is in class $\mathscr{C}\left(\alpha_{2}\right)$ for $\gamma \leq 17 / 6$, where $\alpha_{2}=1-(6 / 17) \gamma$.

(iii) For $v=3 / 2$, the function

$$
\begin{aligned}
G_{3 / 2, \gamma}(z)=\int_{0}^{z}\left(\mathscr{K}_{3 / 2}^{\prime}(t)\right)^{\gamma} d t=\int_{0}^{z}\left(\frac{-8}{t^{2}}\right. \\
-8\left(\frac{\cos (\sqrt{t})}{2 t}-\frac{\sin (\sqrt{t})}{2 t \sqrt{t}}-\frac{\sin (\sqrt{t})}{2 t}\right. \\
\left.\left.-\frac{\cos (\sqrt{t})}{2 t \sqrt{t}}\right)\right)^{\gamma} d t
\end{aligned}
$$

is in class $C\left(\alpha_{3}\right)$ for $\gamma \leq 29 / 6$, where $\alpha_{3}=1-(6 / 29) \gamma$.
3.2. Modified Struve Functions. We obtain the modified Struve function of first kind of order $v$, denoted by $L_{v}(z)$, defined by (9), by putting $b=-c=1$ in (11). Define a function $\mathscr{L}_{v}(z): \mathscr{U} \rightarrow \mathbb{C}$ by

$$
\mathscr{L}_{v}(z)=2^{v} \sqrt{\pi} \Gamma\left(v+\frac{3}{2}\right) z^{(-1-v) / 2} L_{v}(\sqrt{z}) .
$$

We observe that

$$
\mathscr{L}_{1 / 2}(z)=2(\cosh \sqrt{z}-1) .
$$

By making use of this particular value, we have the following assertions.

Corollary 6. Let $v_{1}, \ldots, v_{n}>-1.75$ where $n \in \mathbb{N}$. Consider the function $\mathscr{L}_{v}(z): \mathcal{U} \rightarrow \mathbb{C}$ defined by

$$
\mathscr{L}_{v_{i}}(z)=2^{v_{i}} \sqrt{\pi} \Gamma\left(v_{i}+\frac{3}{2}\right) z^{\left(\left(-v_{i}-1\right) / 2\right)} L_{v_{i}}(\sqrt{z}),
$$

and $v=\min \left\{v_{1}, v_{2}, \ldots, v_{n}\right\}$ and $\gamma_{1}, \gamma_{2}, \ldots, \gamma_{n}$ be positive real numbers. Suppose that these numbers satisfy the inequality

$$
0 \leq 1-\frac{4(3 v+4)}{3\left(24 v^{2}+58 v+35\right)} \sum_{i=1}^{n} \gamma_{i}<1,
$$

then the function $W_{v_{i}, \gamma_{i}}(z): \mathcal{U} \rightarrow \mathbb{C}$ defined as

$$
W_{v_{i}, \gamma_{i}}(z)=\int_{0}^{z} \prod_{i=1}^{n}\left(\frac{\mathscr{L}_{v_{i}}(t)}{t}\right)^{\gamma_{i}} d t
$$

is in class $\mathscr{C}(\alpha)$, where

$$
\alpha=1-\frac{4(3 v+4)}{3\left(24 v^{2}+58 v+35\right)} \sum_{i=1}^{n} \gamma_{i} .
$$

(i) In particular for $v=1 / 2$, the integral operator

$$
\begin{aligned}
W_{1 / 2, \gamma}(z) & =\int_{0}^{z}\left(\frac{\mathscr{L}_{1 / 2}(t)}{t}\right)^{\gamma} d t \\
& =\int_{0}^{z}\left(\frac{2 \cosh \sqrt{t}-1}{t}\right)^{\gamma} d t
\end{aligned}
$$

is in class $C\left(\alpha_{1}\right)$ for $\gamma \leq 105 / 11$, where $\alpha_{1}=1-(11 / 105) \gamma$.

Corollary 7. Let $v_{1}, \ldots, v_{n}>-1.75$, where $n \in \mathbb{N}$. Consider the function $\mathscr{L}_{v}(z): \mathscr{U} \rightarrow \mathbb{C}$ defined by

$$
\mathscr{L}_{v_{i}}(z)=2^{v_{i}} \sqrt{\pi} \Gamma\left(v_{i}+\frac{3}{2}\right) z^{\left(\left(-v_{i}-1\right) / 2\right)} L_{v_{i}}(\sqrt{z}),
$$

$v=\min \left\{v_{1}, v_{2}, \ldots, v_{n}\right\}$, and $\gamma_{1}, \gamma_{2}, \ldots, \gamma_{n}$ be positive real numbers. Suppose that these numbers satisfy the relation

$$
0 \leq 1-\frac{6}{12 v+11} \sum_{i=1}^{n} \gamma_{i}<1,
$$

then the function $G_{v_{i}, \gamma_{i}}(z): \mathscr{U} \rightarrow \mathbb{C}$ defined as

$$
G_{v_{i}, \gamma_{i}}(z)=\int_{0}^{z} \prod_{i=1}^{n}\left(\mathscr{L}_{v_{i}}^{\prime}(t)\right)^{\gamma_{i}} d t
$$


is $\mathscr{C}(\alpha)$, where

$$
\alpha=1-\frac{6}{12 v+11} \sum_{i=1}^{n} \gamma_{i}
$$

In particular for $v=1 / 2$, the function

$$
G_{1 / 2, \gamma}(z)=\int_{0}^{z}\left(\mathscr{L}_{1 / 2}^{\prime}(t)\right)^{\gamma} d t=\int_{0}^{z}\left(\frac{\sinh (\sqrt{t})}{\sqrt{t}}\right)^{\gamma} d t
$$

is in class $\mathscr{C}\left(\alpha_{1}\right)$ for $\gamma \leq 17 / 6$, where $\alpha_{1}=1-(6 / 17) \gamma$.

\section{Locally Univalence Criteria}

Now, we find the locally univalent criteria of the integral operators involving generalized Struve functions defined in (15), (16).

Theorem 8. Let $v_{1}, \ldots, v_{n}, b \in \mathbb{R}$ and $c \in \mathbb{C}$ such that $l_{i}>$ $7|c| / 24$, where $l_{i}=v_{i}+(b+2) / 2(i=1, \ldots, n)$. Consider the function $u_{v_{i}, b, c}: \mathscr{U} \rightarrow \mathbb{C}$, defined as

$$
u_{v_{i}, b, c}(z)=2^{v_{i}} \sqrt{\pi} \Gamma\left(v_{i}+\frac{b+2}{2}\right) z^{\left(\left(-1-v_{i}\right) / 2\right)} w_{v_{i}, b, c}(z) .
$$

Also suppose that $l=\min \left\{l_{1}, l_{2}, \ldots, l_{n}\right\}$ and $\gamma_{1}, \gamma_{2}, \ldots, \gamma_{n}$ be positive real number and that satisfies the inequality

$$
0<\frac{8|c|}{12 l-7|c|} \sum_{i=1}^{n} \gamma_{i} \leq 1
$$

then $G_{v_{i}, b, c, \gamma_{i}}(z): \mathscr{U} \rightarrow \mathbb{C}$ defined in (16) is in class $\mathscr{G}(\beta)$, where

$$
\beta=\frac{8|c|}{12 l-7|c|} \sum_{i=1}^{n} \gamma_{i}
$$

Proof. Differentiating (16), we obtain

$$
G_{v_{i}, b, c, \gamma_{i}}(z)=\prod_{i=1}^{n}\left(u_{v_{i}, b, c}^{\prime}(z)\right)^{\gamma_{i}} .
$$

Differentiating logarithmically, we obtain

$$
\mathfrak{R}\left(1+\frac{z G_{v_{i}, b, c, \gamma_{i}}^{\prime \prime}(z)}{G_{v_{i}, b, c, \gamma_{i}}^{\prime}(z)}\right)=\sum_{i=1}^{n} \gamma_{i} \mathfrak{R}\left(\frac{z u_{v_{i}, b, c}^{\prime \prime}(z)}{u_{v_{i}, b, c}^{\prime}(z)}\right)+1 .
$$

By using (ii) of Lemma 1, it follows that

$$
\mathfrak{R}\left\{\frac{z u_{v, b, c}^{\prime \prime}(z)}{u_{v, b, c}^{\prime}(z)}\right\} \leq \frac{6|c|}{12 l-7|c|} .
$$

Therefore, we have

$$
\begin{aligned}
& \Re\left(1+\frac{z G_{v_{i}, b, c, \gamma_{i}}^{\prime \prime}(z)}{G_{v_{i}, b, c, \gamma_{i}}^{\prime}(z)}\right) \leq \sum_{i=1}^{n} \gamma_{i}\left(\frac{6|c|}{12 l_{i}-7|c|}\right)+1 \\
& \quad<1+\frac{2 \sum_{i=1}^{n} \gamma_{i}\left(6|c| /\left(12 l_{i}-7|c|\right)\right)}{2} \\
& \quad<1+\frac{\sum_{i=1}^{n} \gamma_{i}\left(8|c| /\left(12 l_{i}-7|c|\right)\right)}{2} .
\end{aligned}
$$

For $z \in \mathscr{U}$ and $l_{i}>(7|c| / 24) \forall i=1, \ldots, n$, we consider the function

$$
\Phi:\left(\frac{7|c|}{24}, \infty\right) \rightarrow \mathbb{R}
$$

defined by

$$
\Phi(x)=\frac{1}{2} \frac{8|c|}{12 x-7|c|}
$$

is decreasing $\forall i=1, \ldots, n$; therefore

$$
\frac{1}{2} \frac{8|c|}{12 l_{i}-7|c|} \leq \frac{1}{2} \frac{8|c|}{12 l-7|c|} .
$$

This implies that

$$
\mathfrak{R}\left(1+\frac{z G_{v_{i}, b, c, \gamma_{i}}^{\prime \prime}(z)}{G_{v_{i}, b, c, \gamma_{i}}^{\prime}(z)}\right)<1+\frac{1}{2} \frac{8|c|}{12 l-7|c|} \sum_{i=1}^{n} \gamma_{i} .
$$

Let

$$
0 \leq \frac{8|c|}{12 l-7|c|} \sum_{i=1}^{n} \gamma_{i}<1
$$

Hence

$$
\Re\left(1+\frac{z G_{t_{i}, a, b, r_{i}}^{\prime \prime}(z)}{G_{t_{i}, a, b, \gamma_{i}}^{\prime}(z)}\right)<1+\frac{\beta}{2} .
$$

Here $\beta=8|c| /(12 l-7|c|) \sum_{i=1}^{n} \gamma_{i}$, which completes the proof.

Theorem 9. Let $v_{1}, \ldots, v_{n}, b \in \mathbb{R}$ and $c \in \mathbb{C}$ be such that $l_{i}>$ $7|c| / 24$, where $l_{i}=v_{i}+(b+2) / 2(i=1, \ldots, n)$. Consider the function $u_{v_{i}, a, b}: \mathcal{U} \rightarrow \mathbb{C}$, defined as

$$
u_{v_{i}, b, c}(z)=2^{v_{i}} \sqrt{\pi} \Gamma\left(v_{i}+\frac{b+2}{2}\right) z^{\left(\left(-1-v_{i}\right) / 2\right)} w_{v_{i}, b, c}(z) .
$$

Also suppose that $l=\min \left\{l_{1}, l_{2}, \ldots, l_{n}\right\}$ and $\gamma_{1}, \gamma_{2}, \ldots, \gamma_{n}$ are positive real number and that satisfies the inequality

$$
0<\frac{2|c|(6 l-|c|)}{3(4 l-|c|)(3 l-|c|)} \sum_{i=1}^{n} \gamma_{i} \leq 1,
$$

then $W_{v_{i}, b, c, \gamma_{i}}(z): \mathscr{U} \rightarrow \mathbb{C}$ defined in (15) is in class $\mathscr{G}(\beta)$, where

$$
\beta=\frac{2|c|(6 l-|c|)}{3(4 l-|c|)(3 l-|c|)} \sum_{i=1}^{n} \gamma_{i}
$$

Proof. Differentiating (15), we have

$$
W_{v_{i}, b, c, \gamma_{i}}^{\prime}(z)=\sum_{i=1}^{n}\left(\frac{u_{v_{i}, b, c}(z)}{z}\right)^{\gamma_{i}}
$$

Differentiating logarithmically, we obtain

$$
\begin{aligned}
& \mathfrak{R}\left(1+\frac{z W_{v_{i}, b, c, \gamma_{i}}^{\prime \prime}(z)}{W_{v_{i}, b, c, \gamma_{i}}^{\prime}(z)}\right) \\
& =\sum_{i=1}^{n} \gamma_{i} \mathfrak{R}\left(\frac{z u_{v_{i}, b, c}^{\prime}(z)}{u_{v_{i}, b, c}(z)}\right)+\left(1-\sum_{i=1}^{n} \gamma_{i}\right) .
\end{aligned}
$$


Now consider (i) of Lemma 1, we obtain

$$
\mathfrak{R}\left\{\frac{z u_{v, b, c}^{\prime}(z)}{u_{v, b, c}(z)}\right\}<1+\frac{|c|(6 l-|c|)}{3(4 l-|c|)(3 l-|c|)} .
$$

Therefore

$$
\begin{aligned}
& \mathfrak{R}\left(1+\frac{z W_{v_{i}, b, c, \gamma_{i}}^{\prime \prime}(z)}{W_{v_{i}, b, c, \gamma_{i}}^{\prime}(z)}\right) \\
& \quad \leq \sum_{i=1}^{n} \gamma_{i}\left(1+\frac{|c|\left(6 l_{i}-|c|\right)}{3\left(4 l_{i}-|c|\right)\left(3 l_{i}-|c|\right)}\right)+1-\sum_{i=1}^{n} \gamma_{i} .
\end{aligned}
$$

For $z \in \mathscr{U}$ and $l_{i}>(7|c| / 24) \forall i=1, \ldots, n$, we consider the function

$$
\Lambda:\left(\frac{7|c|}{24}, \infty\right) \rightarrow \mathbb{R}
$$

defined by

$$
\Lambda(x)=\frac{1}{2} \frac{2|c|(6 x-|c|)}{3(4 x-|c|)(3 x-|c|)}
$$

therefore

$$
\begin{gathered}
\frac{1}{2} \sum_{i=1}^{n} \gamma_{i}\left(\frac{2|c|\left(6 l_{i}-|c|\right)}{3\left(4 l_{i}-|c|\right)\left(3 l_{i}-|c|\right)}\right) \\
\quad \leq \frac{1}{2} \frac{2|c|(6 l-|c|)}{3(4 l-|c|)(3 l-|c|)} \sum_{i=1}^{n} \gamma_{i} .
\end{gathered}
$$

This implies that

$$
\begin{aligned}
& \Re\left(1+\frac{z W_{v_{i}, b, c, \gamma_{i}}^{\prime \prime}(z)}{W_{v_{i}, b, c, \gamma_{i}}^{\prime}(z)}\right) \\
& \quad<1+\frac{(2|c|(6 l-|c|) / 3(4 l-|c|)(3 l-|c|)) \sum_{i=1}^{n} \gamma_{i}}{2} .
\end{aligned}
$$

Let

$$
0 \leq \frac{2|c|(6 l-|c|)}{3(4 l-|c|)(3 l-|c|)} \sum_{i=1}^{n} \gamma_{i}<1 .
$$

Hence

$$
\mathfrak{R}\left(1+\frac{z W_{v_{i}, b, c, \gamma_{i}}^{\prime \prime}(z)}{W_{v_{i}, b, c, \gamma_{i}}^{\prime}(z)}\right)<1+\frac{\beta}{2} .
$$

Here $\beta=2|c|(6 l-|c|) / 3(4 l-|c|)(3 l-|c|) \sum_{i=1}^{n} \gamma_{i}$, which completes the proof.

\section{Conflicts of Interest}

The authors declare that there are no conflicts of interest regarding the publication of this paper.

\section{Acknowledgments}

The authors would like to acknowledge the heads of their institutions for their supportive role and research facilities. This research is conducted under the Project of Higher Education Commission of Pakistan (Project Ref: 5689/Punjab/NRPU/R\&D/2016) and is partially supported by Sarhad University of Science and Information Technology, Peshawar, Pakistan.

\section{References}

[1] S. Ozaki, "On the theory of multivalent functions II," Science Reports of the Tokyo Bunrika Daigaku, Section A, vol. 4, pp. 4586, 1941.

[2] M. Obradović, S. Ponnusamy, and K. J. Wirths, "Coefficient characterizations and sections for some univalent functions," Siberian Mathematical Journal, vol. 54, no. 4, pp. 679-696, 2013.

[3] R. Kargar and A. Ebadian, "Ozakis conditions for general integral operator," Sahand Comm. Math. Anal, vol. 5, no. 1, pp. 61-67, 2017.

[4] M. Nunokawa and J. Sokół, "An improvement of Ozaki’s condition," Applied Mathematics and Computation, vol. 219, no. 22, pp. 10768-10776, 2013.

[5] S. Zhang and J. Jin, Computation of special functions, Wiley Interscience Publication, New York, NY, USA, 1996.

[6] H. Orhan and N. Yagmur, "Geometric properties of generalized Struve functions," Analele Ştiinţifice ale Universităţii "Al. I. Cuza" din Iași. Serie Nouă. Matematică. Annals of the Alexandru Cuza University. Mathematics, vol. 63, no. 2, pp. 229-244, 2017.

[7] A. Baricz, D. K. Dimitrov, H. Orhan, and N. Yagmur, "Radii of starlikeness of some special functions," Proceedings of the American Mathematical Society, vol. 144, no. 8, pp. 3355-3367, 2016.

[8] H. Orhan and N. Yagmur, "Starlikeness and convexity of generalized Struve functions," Abstract and Applied Analysis, vol. 2013, Article ID 954513, 6 pages, 2013.

[9] M. Raza and N. Yagmur, "Some properties of a class of analytic functions defined by generalized Struve functions," Turkish Journal of Mathematics, vol. 39, no. 6, pp. 931-944, 2015.

[10] M. Din, H. M. Srivastava, and M. Raza, "Univalence of certain integral operators involving generalized Struve functions," Hacett. J. Math. Stat, 2017.

[11] M. Abramowitz and I. A. Stegun, Handbook of Mathematical Functions, Dover, NY, USA, 1965. 


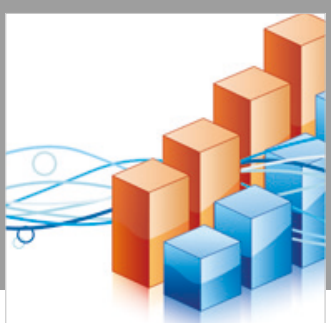

Advances in

Operations Research

\section{-n-m}
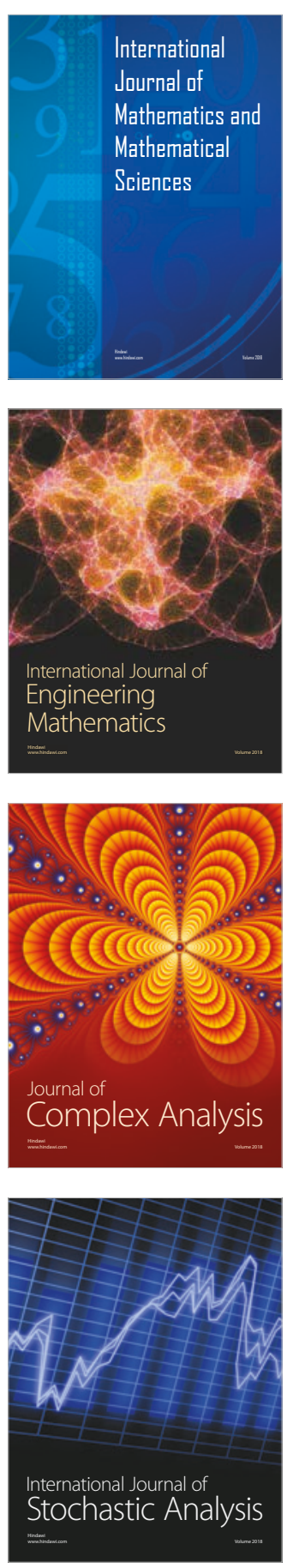
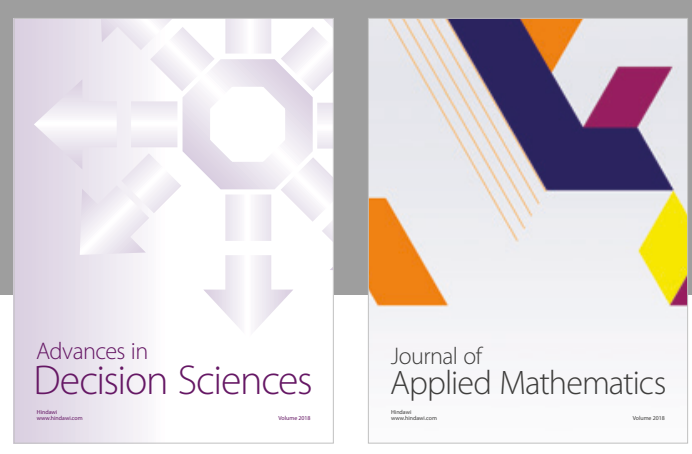

Journal of

Applied Mathematics
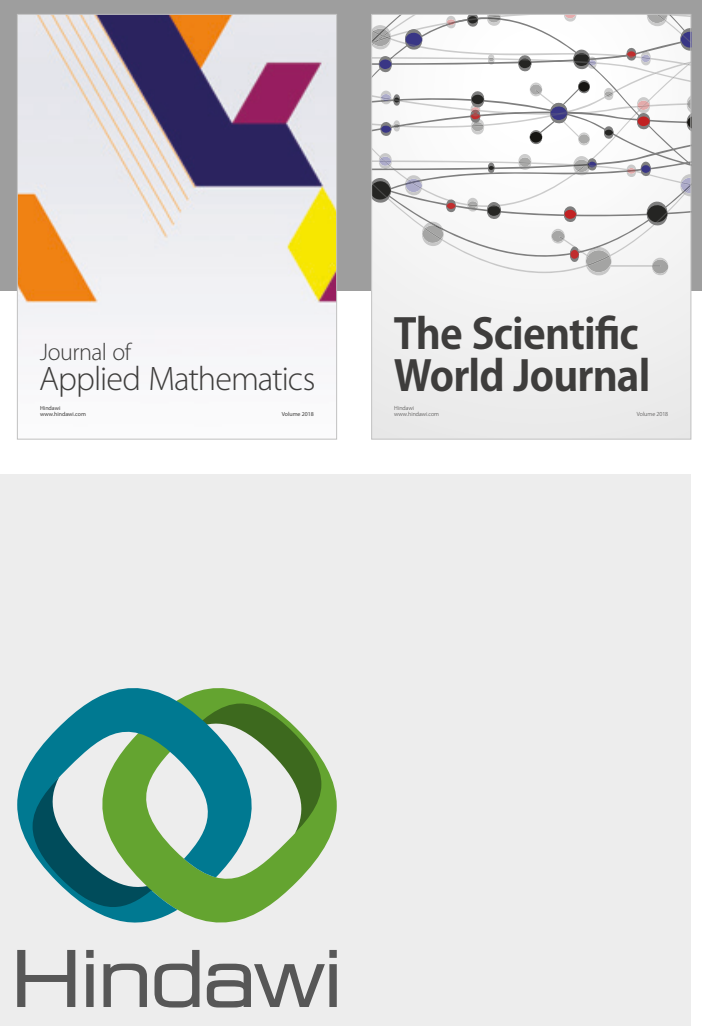

Submit your manuscripts at

www.hindawi.com

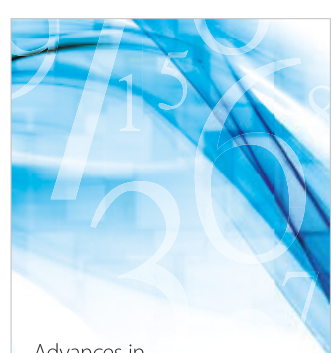

Advances in
Numerical Analysis
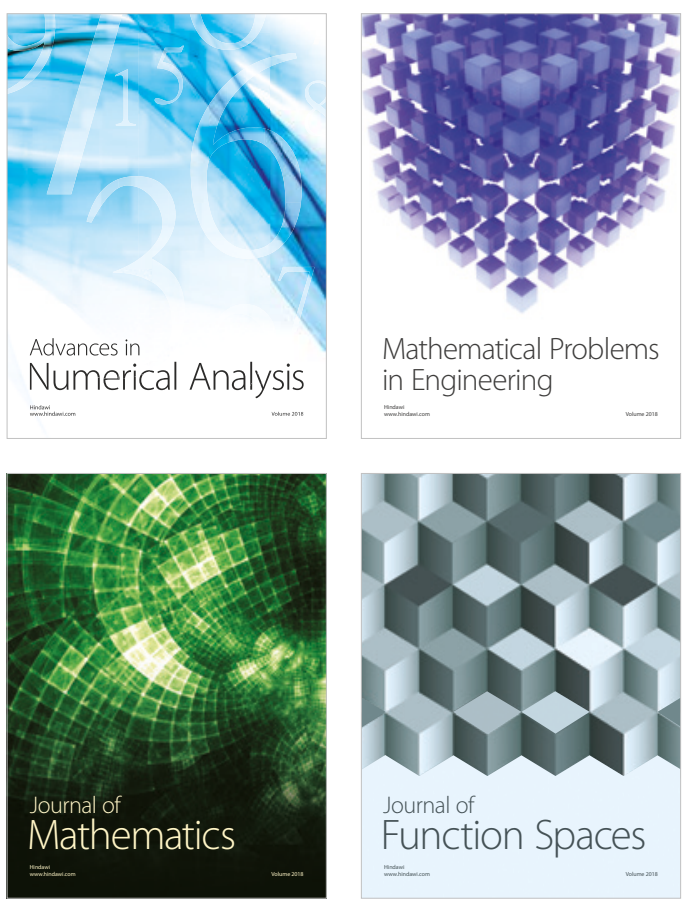

Mathematical Problems in Engineering

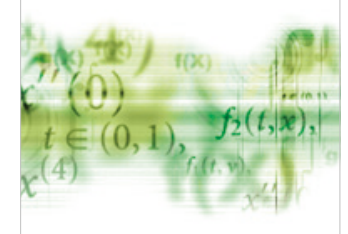

International Journal of

Differential Equations

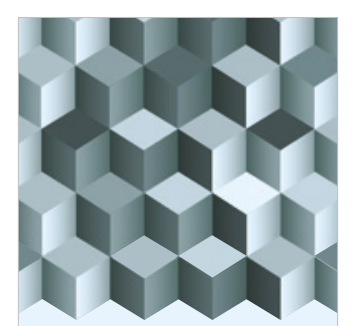

Journal of

Function Spaces

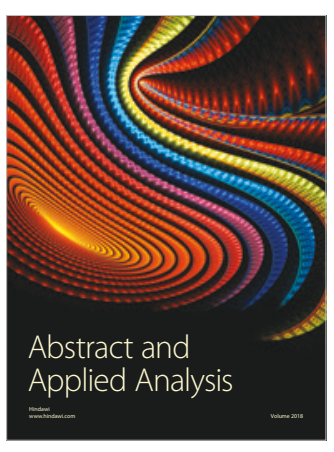

The Scientific

World Journal

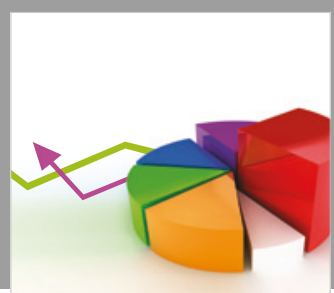

Journal of

Probability and Statistics
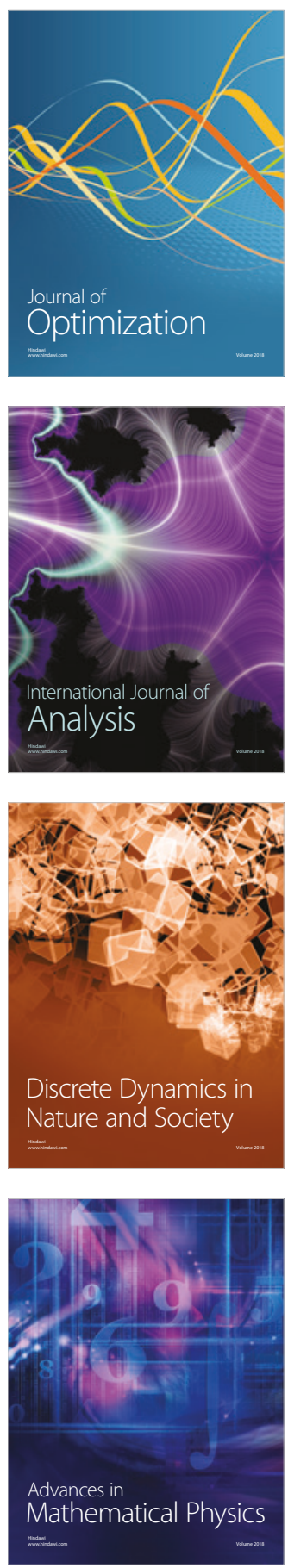